\title{
Computer-Aided Thyroid Nodule Detection in Ultrasound Images
}

\author{
D.E. Maroulis ${ }^{\mathrm{a}}$, M.A. Savelonas ${ }^{\mathrm{a}}$, S.A. Karkanis ${ }^{\mathrm{b}}$, D.K. Iakovidis ${ }^{\mathrm{a}}$, N. Dimitropoulos ${ }^{\mathrm{c}}$ \\ ${ }^{a}$ Dept. of Informatics and Telecommunication, University of Athens, Greece \\ ${ }^{\mathrm{b}}$ Dept. of Informatics and Computer Technology, Technological Educational Institute \\ of Lamia, Greece \\ ${ }^{\mathrm{c}}$ Dept. of Medical Imaging, Euromedica Medical Center, Athens, Greece \\ rtsimage@di.uoa.gr
}

\begin{abstract}
Nodular thyroid disease is a frequent occurrence in clinical practice and it is associated with increased risk of thyroid cancer and hyperfunction. In this paper we propose a novel method for computer-aided detection of thyroid nodules in ultrasound (US) images. The proposed method is based on a level-set image segmentation approach that takes into account the inhomogeneity of the US images. This novel method was experimentally evaluated using US images acquired from 35 patients. The results show that the proposed method achieves more accurate delineation of the thyroid nodules in the US images and faster convergence than other relevant methods.
\end{abstract}

\section{Introduction}

Nodular thyroid disease is extremely common and of concern because of the risk of malignancy and hyperfunction. The risk of developing a palpable thyroid nodule in a lifetime ranges between 5 and $10 \%$, while $50 \%$ of people with solitary nodules detected by experienced physicians have additional nodules detected when examined further by ultrasonography [1].

Thyroid ultrasonography is a non-invasive diagnostic test, which provides immediate information on the structure and the characteristics of thyroid nodules. It combines low cost, short acquisition time, absence of ionizing radiations and sensitivity in ascertaining the size and number of thyroid nodules. However ultrasound (US) images contain echo perturbations and speckle noise, which could make the diagnostic task harder. Additionally, image interpretation, as performed by the experts, is subjective. Therefore, a method for computeraided thyroid nodule detection should take into consideration the inherent noise characteristics of the US images and be capable of interpreting these images, based on explicit image features. Such a method could contribute to the objectification of the medical diagnosis and consequently to a reduction of false decisions.

Active contour models first appeared in the late eighties [2]. The classic active contour approach in image segmentation is based on the deformation of initial contours towards the boundaries of the image regions to be segmented. The deformation is realized by the minimization of an energy functional designed so that its local minimum is reached at the target boundaries. The energy functional in its basic form is comprised of two components, the first controls the smoothness of the contour and the second is image dependent and forces the contour towards the boundary. This active contour approach is boundary based and utilizes local filtering techniques such as edge detection operators. In the case of noisy images, such as US images, many unwanted edges may appear due to noise, and should consequently be smoothed by the application of a strong isotropic Gaussian filter. Such filtering introduces the risk of smoothing the target boundaries and therefore contour leakage effects may appear resulting in diminution of the delineation accuracy [3]. Moreover, the 
parametric formulation of the classic active contour approach, does not allow for changes in the topology of the evolving contour, such as splitting and merging. Therefore, complementary procedures have to be considered to enable adaptability to the required topological changes [4].

Active contours have been employed in various medical US image analysis applications either in parametric or in level set form. Parametric active contour applications include the detection of hepatic tumors [5], the detection of lumen and media-adventitia border in sequential intravascular ultrasound (IVUS) frames [6] and the evaluation of margins for malignant breast tumor excision through mammotomes [7]. Level set active contour applications include the automatic quantification of the ventricular function [8] and the segmentation of prostate [9] and cardiac US images [10]. To the best of our knowledge there has not been proposed any information technology approach to thyroid nodule detection in US images.

Active Contours Without Edges (ACWE) [3] has been proposed as a noise-robust image segmentation method. It is capable of detecting objects even with smooth boundaries due to its region-based approach in which the functional is a combination of domain and boundary integrals. Moreover, following the level set formulation, originally proposed in [11], it is capable of detecting two or more objects in the image as it provides adaptability to topological changes e.g. contour splitting. A limitation of this model is that it presumes homogeneity for object and background areas. This presumption is violated in thyroid US images due to the intensity inhomogeneity of the thyroid tissue texture and the presence of calcifications appearing in the form of bright spots. A modification that takes into account image inhomogeneity could lead to more accurate object detection.

In this paper, we propose a novel level set active contour model, for thyroid nodule detection in US images that takes into account image inhomogeneity by utilizing a variable background approach for the enhancement of the nodule detection accuracy while achieving faster contour convergence.

The rest of this paper is organized in three sections. Section 2 includes a brief description of the Active Contour Without Edges model and the presentation of the proposed Variable Background Active Contour model. The experimental results from the application of the proposed model on thyroid ultrasound images are apposed in Section 3. Finally, Section 4 summarizes the conclusions of this study.

\section{Variable Background Active Contour Model}

\subsection{Active Contour Without Edges}

The ACWE model as posed in [3] has the form of a minimization problem: Let $\Omega$ be a bounded open subset of $R^{2}$ and $\partial \Omega$ its boundary. We seek for inf $F\left(c^{+}, c^{-}, C\right)$,

$$
\begin{aligned}
F\left(c^{+}, c^{-}, C\right) & =\mu \cdot \text { Length }(C) \\
& +\lambda^{+} \int_{\substack{\text { inside }(C)\\
}}\left|u_{0}(x, y)-c^{+}\right|^{2} d x d y \\
& +\lambda^{-} \int_{\substack{\text { outside }(C) \\
\text { ( })}}\left|u_{0}(x, y)-c^{-}\right|^{2} d x d y
\end{aligned}
$$

where $u_{0}: \Omega \rightarrow R$ is the input image, $C(s):[0,1] \rightarrow R^{2}$ a piecewise parameterized curve, $c^{+}$and $c^{-}$are unknown constants representing the average value of $u_{0}$ inside and outside the curve and parameters $\mu>0$ and $\lambda^{+}, \lambda^{-}>0$ are weights for the regularizing 
term and the fitting terms, respectively. This formulation describes a special case of the minimal partition problem, for which the existence of minimizers has been proved in [12] for $u_{0}$ continuous on $\Omega$ and in [13] for more general data. As in the minimum energy problem, the minimizer corresponds to the "equilibrium" of the regularizing and fitting terms that force the contour to stop. It should be noted that, as implied by (1), this model assumes that the image is formed by two regions of approximately piecewise constant intensities.

In the level set method [11], $C \subset \Omega$ is represented by the zero level set of a Lipschitz function $\phi: \Omega \rightarrow R$, such that

$$
\begin{aligned}
& C=\{(x, y) \in \Omega: \phi(x, y)=0\}, \\
& \text { inside }(C)=\{(x, y) \in \Omega: \phi(x, y)>0\}, \\
& \text { outside }(C)=\{(x, y) \in \phi(x, y)<0\}
\end{aligned}
$$

Using the one-dimensional Dirac measure $\delta$ and the Heaviside function $H$, which are defined respectively by

$$
\delta(z)=\frac{d}{d z} H(z), H(z)= \begin{cases}1, & \text { if } z \geq 0 \\ 0, & \text { if } z<0\end{cases}
$$

where $\mathrm{z} \in R$, the constants $c^{+}$and $c^{-}$can be expressed as

$$
\begin{gathered}
c^{+}(\phi)=\frac{\int_{\Omega} u_{0}(x, y) H(\phi(x, y)) d x d y}{\int_{\Omega} H(\phi(x, y)) d x d y} \\
c^{-}(\phi)=\frac{\int_{\Omega} u_{0}(x, y)(1-H(\phi(x, y))) d x d y}{\int_{\Omega}(1-H(\phi(x, y))) d x d y}
\end{gathered}
$$

By keeping $c^{+}$and $c^{-}$fixed, and minimizing $F$ with respect to $\phi$, the associated Euler-Langrange equation for $\phi$ is deduced. For this purpose, slightly regularized versions of $H$ and $\delta$ are considered. The applied $C^{\infty}(\overline{\Omega)}$ regularized Heaviside function $H_{\varepsilon}$ is derived from

$$
H_{\varepsilon}=\frac{1}{2} \cdot\left(1+\frac{2}{\pi} \arctan \left(\frac{z}{\varepsilon}\right)\right)
$$

whereas the corresponding regularized delta function $\delta_{\varepsilon}$ is derived from $\delta_{\varepsilon}=d H_{\varepsilon} / d z$. As $\varepsilon \rightarrow 0$, both approximations converge to $H$ and $\delta$. These approximations allow the algorithm to compute a global minimizer, as described in [3].

Parameterizing the descent direction by an artificial time $t \geq 0$, the equation in $\phi(t, x, y)$ (with $\phi(0, x, y)=\phi_{0}(x, y)$ defining the initial contour) is

$$
\frac{\partial \phi}{\partial t}=\delta(\phi)\left[\mu \cdot \operatorname{div}\left(\frac{\nabla \phi}{|\nabla \phi|}\right)-\lambda^{+}\left(u_{0}-c^{+}\right)^{2}+\lambda^{-}\left(u_{0}-c^{-}\right)^{2}\right]=0
$$


where $t \in(0, \infty),(x, y) \in \Omega$.

In a practical implementation, a quantitative criterion should force the algorithm to stop when the changes of $\phi$ fall bellow a threshold for a fixed number of iterations. When this criterion is satisfied it is assumed that the minimizer is found and the corresponding equilibrium has been reached.

\subsection{Variable Background Active Contour}

The proposed model, named Variable Background Active Contour (VBAC) follows Eq. (1) where $c^{-}$is derived from Eq. (8):

$$
c^{-}(\phi)=\frac{\int_{\Omega} u_{0}(x, y)(1-H(\phi(x, y))) H\left(\phi_{0}(x, y)\right) \Delta(x, y) d x d y}{\int_{\Omega}(1-H(\phi(x, y))) H\left(\phi_{0}(x, y)\right) \Delta(x, y) d x d y}
$$

and the difference term $\Delta(x, y)$ is given by:

$$
\Delta(x, y)=H(\phi(x, y)-a)-H(\phi(x, y)), \quad a>0
$$

Note that $H\left(\phi_{0}\right)$ restricts the integrals in a region of interest, which for the purposes of our study coincides with the thyroid gland. The constant $a$, determines the background area considered. The introduction of $\Delta(x, y)$ reduces the effects of background area inhomogeneities corresponding to calcifications appearing in the US image in the form of bright spots as well as to the intensity inhomogeneity of the thyroid tissue texture. This can be justified by the fact that these inhomogeneities cause abrupt changes of $\phi$ which result in $H(\phi(x, y)-a)=H(\phi(x, y))=1$ for the inhomogeneous areas in the image. Therefore, $\Delta(x, y) \neq 0$ is satisfied in a limited image subset, which excludes inhomogeneous areas.

\section{Results}

Thyroid ultrasound examinations were performed on 35 patients using a digital ultrasound system HDI 3000 ATL with a 5-12 MHz linear transducer. The acquired digital images had a resolution of $256 \times 256$ pixels and 256 gray-level depth. We developed a special purpose software suite in Microsoft Visual C++ for the implementation of the ACWE and the VBAC models. Both of these models were applied for thyroid nodule detection in the US images using $\lambda^{+}=5, \lambda^{-}=5, \mu=650$ and $a=10^{-13}$. For the purposes of our study we adopted the image intensity as the supervising feature for the contour evolution, to enable the detection of hypo-echoic thyroid nodules. Low echogenicity characterizes the majority of thyroid nodules and especially those that are suspect of malignancy [14].

Three expert radiologists manually delineated the thyroid nodules to enable comparisons with the active contour models. For each US image, "ground truth" delineation is obtained, following the rule that each pixel is considered as part of the nodule when it is included in at least two out of three experts' delineations [15]. As a measure of similarity between a delineated area $A$ and the "ground truth" delineated area $G$, we have considered the overlap value [16]:

$$
i=\frac{A \cap G}{A \cup G}
$$


The inter-observer variation was estimated $7.4 \%$ in terms of the Coefficient of Variation $\mathrm{CV} \%=100 *\left(\sigma_{i} / m_{i}\right)$, where $\sigma_{i}$ and $\mathrm{m}_{i}$ are the standard deviation and the mean value of $i$, respectively [15].

The experiments showed that the proposed VBAC model and the ACWE model converged to overlap values with an average difference of $\Delta i=i_{V B A C}-i_{A C W E}=6.2 \pm 2.3 \%$. Both models resulted in a maximum overlap of $99.1 \%$. The minimum overlap values obtained were $78.6 \%$ and $62.1 \%$ for the VBAC and the ACWE models respectively, whereas the corresponding mean overlap values were $88.8 \%$ and $82.6 \%$. Moreover with the VBAC model the convergence was reached in $10 \%$ less algorithm iterations than with the ACWE model, which is translated in approximately $8.5 \%$ speedup in terms of absolute execution time.

Figure 1 illustrates an example US image delineated by an expert radiologist, the VBAC and the ACWE models respectively. It can be observed that the VBAC surrounded the hypoechoic nodule more accurately than the ACWE compared to the expert's delineation. The overlap values achieved per iteration for the image of Fig. 1 are illustrated in Fig. 2. This figure shows that the VBAC model converges to a higher overlap value (94.6\%) in approximately $10^{3}$ less iterations than the ACWE model which leads to a maximum overlap value of $80.9 \%$.

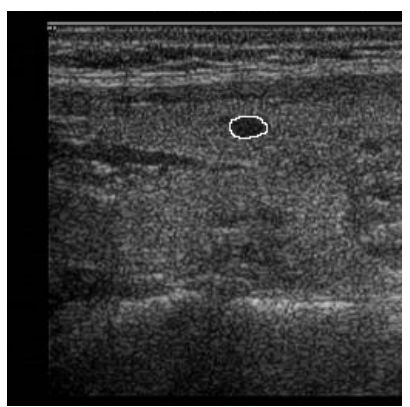

(a)

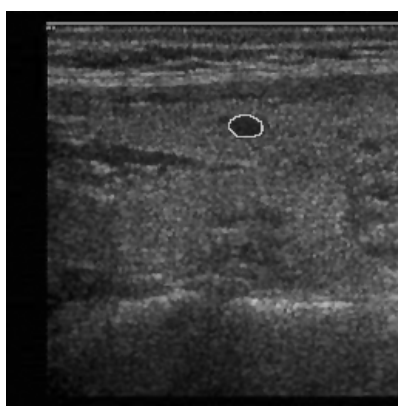

(b)

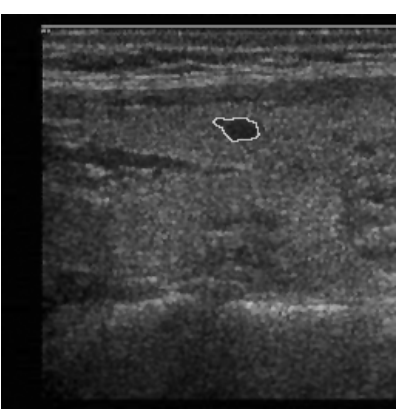

(c)

Figure 1. Two indicative examples of a thyroid nodule in a US image delineated by (a) an expert radiologist, (b) the VBAC, and (c) the ACWE.

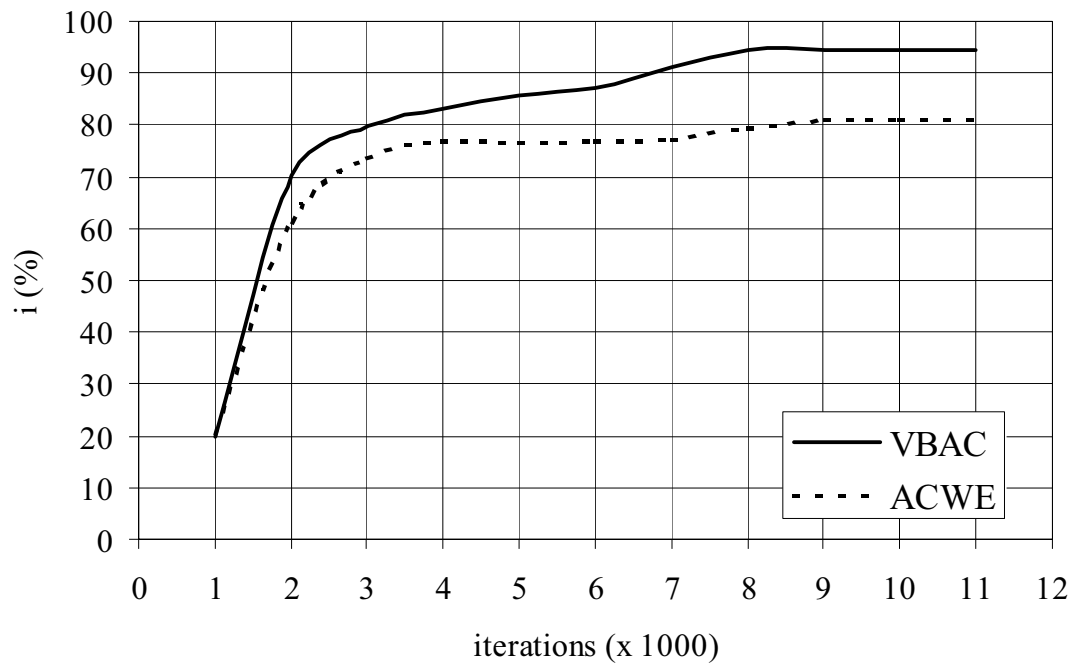

Figure 2. Overlap value $i$ as a function of the number of algorithm iterations corresponding to the US image illustrated in Fig. 1, using the ACWE and the VBAC models. 


\section{Conclusion}

We have proposed a Variable Background Active Contour model and applied it for the detection of thyroid nodules in ultrasound images. In this model, the background is a variable subset of the image, which changes shape to reduce the effects of background inhomogeneity. The results of the experimental study lead to the conclusion that the proposed model provides improved accuracy and faster convergence, compared to the Active Contour Without Edges model. In particular, the improvement in contour accuracy is important due to the fact that nodule size and shape are factors affecting the subsequent nodule classification [1].

Future perspectives of this work include the embedment of textural features to supervise contour evolution enabling the detection of non hypo-echoic nodules.

\section{Acknowledgments}

This work was partially funded by National and Kapodestrian University of Athens, Special Account of Research Grants.

\section{References}

[1] S. Feld et all, "AACE Clinical Practice Guidelines for the Diagnosis and Management of Thyroid Nodules", Endocrine Practice, Jan./Feb. 1996, pp. 78-84.

[2] W. Kass, A. Witkin, D. Terzopoulos, “Snakes: Active Contour Models”, Int. J. Comput. Vision, vol. 1, no. 4, 1987, pp. 321-331.

[3] T.F. Chan, L.A. Vesse, “Active Contours Without Edges”, IEEE Trans. Image Processing, Feb. 2001, pp. 266-277.

[4] T. McInerney, D. Terzopoulos, “Topologically Adaptable Snakes”, Proc. Int. Conf. Comp. Vis., Cambridge, Jun. 1995, pp. 840-845.

[5] C.M. Chen, H.H.S Lu, A.T. Hsiao, "A Dual-Snake Model of High Penetrability for Ultrasound Image Boundary Extraction”, Ultrasound in Med. \& Biol., vol.27, no.12, 2001, pp. 1651-1665.

[6] M.E. Plissiti, D.I. Fotiadis, L.K. Michalis, G.E. Bozios, "An Automated Method for Lumen and MediaAdventitia Border Detection in a Sequence of IVUS Frames", IEEE Trans. On Inf. Tec. in Biomedicine, vol. 8, no. 2, Jun. 2004, pp. 131-141.

[7] R.F. Chang, W.J. Wu, C.C. Tseng, D.R. Chen, W.K. Moon, “ 3-D Snake for US in Margin Evaluation for Malignant Breast Tumor Excision Using Mammotome”, IEEE Trans. On Inf. Tec. in Biomedicine, vol. 7, no. 3, Sep. 2003.

[8] E. Angelini, J. Holmes, A. Laine, "Segmentation of RT3D Ultrasound with Implicit Deformable Models Without Gradients”, Proc. ISPA 2003, Rome, Italy, Sep. 2003, Part II, pp. 711-716.

[9] D. Honigmann, J. Ruisz, and H. Pottmann, "Fast Model Based Segmentation of Ultrasound Data using an Active Image”, Proc. IEEE Int. Symp. on Biomedical Imaging, Washington DC, 2002, pp. 225-228.

[10] N. Lin, W. Yu, J.S. Duncan, "Combinative Multi-scale Level Set Framework for Echocardiographic Image Segmentation”, Medical Image Analysis, vol. 7, 2003, pp. 529-537.

[11] S. Osher, J. Sethian, "Fronts Propagating with Curvature- Dependent Speed: Algorithms Based on the Hamilton-Jacobi Formulations", Journal Of Computational Physics, vol. 79, 1988, pp.12-49.

[12] D. Mumford, J. Shah, "Optimal Approximation by Piecewise Smooth Functions and Associated Variational Problems”, Commun.Pure Appl. Math., vol.42, 1989, pp 577-685.

[13] J.M. Morrel, S. Solimini, "Segmentation of Images by Variational Methods: A Constructive Approach", Madrid, Spain: Revista Matematica Universidad Computense de Madrid, vol.1, 1988, pp.169-182.

[14] E. Papini et al, "Risk of Malignancy in Nonpalpable Thyroid Nodules: Predictive Value of Ultrasound and Color-Doppler Features", The Journal of Clinical Endocrinology \& Metabolism, vol. 87, no.5, 2002, pp. 19411946.

[15] M.R. Kaus, S.K. Warfield, F.A. Jolesz, R. Kikinis, "Segmentation of Meningiomas and Low Grade Gliomas in MRI", Proc. Second International Conference on Medical Image Computing and Computer-Assisted Intervention, Cambridge, England, 1999, pp. 1-10.

[16] X. Hao, C. Bruce, C. Pislaru and J.F. Greeneaf, "A Novel Region Growing Method for segmenting ultrasound images”, IEEE International Ultrasonics Symposium, Oct. 2000. 